\title{
Vegetation Structure, Floristic Composition and Species Diversity of Acacia catechu Bearing Forest Types in Western Himalaya, India
}

\author{
Sanyam* and Vipasha
}

Department of Silviculture and Agroforestry, College of Forestry, Dr. Y.S. Parmar University

of Horticulture and Forestry, Nauni-Solan- 173 230, H.P., India

*Corresponding author

\section{A B S T R A C T}

\begin{tabular}{|l|}
\hline K e y w o r d s \\
$\begin{array}{l}\text { A. catechu, Forest } \\
\text { types, Plantation, } \\
\text { Phytosociology, } \\
\text { SDI, Similarity } \\
\text { index }\end{array}$ \\
\hline Article Info \\
\hline $\begin{array}{l}\text { Accepted: } \\
\text { 17 January } 2018 \\
\text { Available Online: } \\
\text { 10 February } 2018\end{array}$ \\
\hline
\end{tabular}

\section{Introduction}

Forest composition, community structure and diversity patterns are important ecological attributes significantly correlated with prevailing environmental as well as anthropogenic variables (Gairola et al., 2008; Ahmad et al., 2010). The diversity of tree species is fundamental to total forest biodiversity, because trees provide resources and habitats for almost all other forest species (Huang et al., 2003). Vegetation is a key factor in determining the structure of any ecosystem (Bhatt and Purohit, 2009). Within a plant community it determines microclimate, energy budget, photosynthesis, water regimes, surface runoff and soil temperature. The plant community of a region is a function of time and altitude, however, slope, latitude, aspect, rainfall and humidity also play an important role in the formation of plant communities and their composition (Kharkwal et al., 2005). The species diversity, floristic composition and vegetation structure are important to judge the state of natural forests in a region. The local communities residing in and around these forests are dependent upon forests for an array of ecosystem services and values.

Acacia catechu Willd. is a multipurpose 
moderate sized deciduous tree found throughout the greater parts of India. There are 3 varieties of A. catechu viz. catechu, catechuoides and sundra found in India. Catechu (catechu proper) is found in Jammu and Kashmir, Uttar Pradesh and Himachal Pradesh below $1300 \mathrm{~m}$ elevation (Chauhan, 1999). Generally, it forms pure patches of khair forests but it is also found in association with Acacia modesta, Pinus roxburghii, Mallotus philippensis, Dalbergia sissoo, Zizyphus mauritiana, Terminalia bellerica, $T$. chebula, Wendlandia exserta, Butea monosperma, Anogeissus latifolia, Lannea coromandelica and Shorea robusta (Champion and Seth, 1968).

Catechu is a valuable bioresource and is exploited commercially for katha and cutch (tannin) in North India (Luna, 2005). It is equally significant for the rural communities as they are dependent on this tree to fulfill their need of fuel, fodder, building material etc. and hence khair has become an integral part of socio-economic and cultural life of the people inhabiting the lower hills of Himachal Pradesh. The aim of the present study is to generate quantitative information on species diversity in different $A$. catech $u$ bearing forest types so that it can be beneficial for the foresters and villagers to be aware of their present condition.

\section{Materials and Methods}

\section{Study area and data collection}

The experimental area is located in the subtropical zone of Himachal Pradesh. The area is located between $32^{\circ} 19^{\prime}$ to $30^{\circ} 31^{\prime} \mathrm{N}$ latitude and $77^{\circ} 19^{\prime}$ to $75^{\circ} 50^{\prime} \mathrm{E}$ longitude and lies between 375 to $1552 \mathrm{~m}$ above sea level. This zone affected by all three extreme climatic conditions with high temperature in summers, very low in winter and heavy rainfall in rainy season and a mean annual temperature ranging from 21 to $26^{\circ} \mathrm{C}$. The mean annual rainfall varies from 1400 to 1800 $\mathrm{mm}$ and most of which concentrated during July-August.

Six different $A$. catechu bearing forest types viz. Dry Shiwalik Sal forest, Northern Dry Mixed Deciduous forest, Dry Deciduous Scrub forest, Lower Shiwalik Chirpine forest and Dry Riverine forest including one plantation of $A$. catechu were chosen for phytosociological studies. In each forest type, 4 sites were studied and at each site, 4 sample plots were taken. In all, 72 sample plots of size 0.1 ha were taken. The observations were recorded for trees in all sample plots of $10 \mathrm{~m} \times 10 \mathrm{~m}$, while for shrub and herbs (except grasses) plots of size $5 \mathrm{~m} \times 5 \mathrm{~m}$ and $1 \mathrm{~m} \times 1 \mathrm{~m}$ were laid out in each tree sample plot, respectively.

\section{Data analysis}

Relative basal area, relative density and relative frequency were obtained from the percent frequency, density and basal area according to procedure given by Phillips (1959). IVI, was calculated for all species of trees, shrubs and herbs separately. Similarity index (SI) was determined by the method given by Sorenson (1948). Shannon index of general diversity $(\mathrm{H})$ was calculated by using Margalef formulae (Odum 1971).

\section{Results and Discussion}

The data recorded revealed that the plant genetic spectrum comprised of 75 genera with 86 species belonging to 43 families (Table 1) except grasses. In Dry Shivalik Sal forest, 13 tree, 9 shrub and 9 herb species were recorded. While in Northern Dry Mixed Deciduous forest, 18 tree, 12 shrub and 4 herb species were found. In case of Dry Deciduous Scrub forest, 18 tree, 15 shrub and 9 herb species were available whereas, in Lower Shiwalik Chirpine forest, 15 tree, 13 shrub 
and 6 herb species were recorded. In Dry Riverine forest, 16 tree, 11 shrub and 7 herb species were found. While in A. catechu plantations, 12 shrub and 13 herb species were available. The Dry Deciduous Scrub forest and Northern Dry Mixed Deciduous forest had the maximum overall floristic composition while Dry Shivalik Sal forest had the least. In the former two forest types, ample light conditions were present while in latter; presence of thick crown cover of Shorea robusta barred it from reaching forest floor. Lesser number of herb species under Northern Dry Mixed Deciduous forest, Lower Shiwalik Chirpine forest and Dry Riverine forest was due to pine needle-litter deposition on the forest floor which might have restricted germination of herbaceous flora (Gupta et al., 2007) as Pinus roxburghii is one of the dominant species in the sub tropical Himalayan region and grows under hardy conditions which is in line with findings of Mukhia (2011) and Dangwal et al., (2012) for species composition in Chirpine forests in Himalayas. Mueller (2003) opined that overstorey species enhance spatial heterogeneity of soil fertility resulting in change in shrub dynamics. The distribution of species is also regulated by altitude and physiographic regimes (Sharma et al., 2010) and edaphic conditions, slope, aspect and moisture regime (Adhikari et al., 2009).

Vegetational analysis (Table 1) revealed that in Dry Shiwalik Sal forest, the dominant tree species was Shorea robusta>Lannea coromandelica>Acacia catechu. The dominant shrub species are Randia dumetorum $>$ Murraya koenigii $>$ Woodfordia fruticosa. Dominant herbaceous vegetation was Bidens pilosa>Achyranthus aspera $>$ Xanthium strumarium. In Northern Dry Mixed Deciduous forest, the dominant tree species was Acacia catechu>Pinus roxburghii>Bombax ceiba. The dominant shrub species are Celastrus paniculatus> Zizyphus mauritiana>Woodfordia fruticosa.
Dominant herbaceous vegetation comprised of Bidens pilosa> Parthenium hysterophorus > Urena lobata.

In Dry Deciduous Scrub forest, the dominant tree species were Acacia catechu>Pinus roxburghii $>$ Dalbergia sissoo. The dominant shrub species are Randia dumetorum> Zizyphus oxyphylla>Adhatoda vasica. Dominant herbaceous vegetation comprised of Parthenium hysterophorus> Artemisia vulgaris>Achyranthus aspera. In Lower Shiwalik Chirpine forest, the dominant tree species was Pinus roxburghii>Acacia catechu>Bombax ceiba. The dominant shrub species are Zizyphus oxyphylla>Carrisa carandus $>$ Rubus elliptica. Dominant herbaceous vegetation comprised of Bidens pilosa $>$ Achyranthus aspera $>$ Trifolium repens.

In Dry Riverine forest, the dominant tree species was Acacia catechu>Dalbergia sissoo $>$ Pinus roxburghii. The dominant shrub species are Zizyphus mauritiana $>$ Murraya koenigii>Woodfordia fruticosa. Dominant herbaceous vegetation comprised of Parthenium hysterophorus $>$ Bidens pilosa $>$ Cassia tora. In Acacia catechu plantations, the dominant tree species was Acacia catechu. The dominant shrub species are Murraya koenigii $>$ Carissa carandus> Dodonea viscosa. Dominant herbaceous vegetation comprised of Achyranthus aspera> Parthenium hysterophorus $>$ Bidens pilosa.

The Shannon Diversity Index for trees was higher for Northern Dry Mixed Deciduous forest followed by Dry Deciduous Scrub forest, Dry Shiwalik Sal forest, Dry Riverine forest and Lower Shiwalik Chirpine forest (Table 2). The proportion of different species is more in Northern Dry Mixed Deciduous forest and Dry Deciduous Scrub forest as compared to other forest types (Table 1) resulting in high diversity. 
Table.1 Phytosociological studies under different $A$. catechu bearing forest types

\begin{tabular}{|c|c|c|c|c|c|c|c|c|c|c|c|c|c|c|c|c|c|c|c|c|c|c|c|c|c|}
\hline \multirow[b]{2}{*}{ Species } & \multirow[b]{2}{*}{ Family } & \multicolumn{4}{|c|}{ F1 } & \multicolumn{4}{|c|}{ F2 } & \multicolumn{4}{|c|}{ F3 } & \multicolumn{4}{|c|}{ F4 } & \multicolumn{4}{|c|}{ F5 } & \multicolumn{4}{|c|}{ F6 } \\
\hline & & $\mathrm{RF}$ & RD & RDM & IVI & $\mathrm{RF}$ & RD & RDM & IVI & $\mathrm{RF}$ & RD & RDM & IVI & $\mathrm{RF}$ & RD & RDM & IVI & $\mathrm{RF}$ & RD & RDM & IVI & $\mathrm{RF}$ & RD & RDM & IVI \\
\hline \multicolumn{26}{|c|}{ TREES } \\
\hline $\begin{array}{l}\text { Acacia catechu } \\
\text { Willd. }\end{array}$ & Mimosaceae & 12.50 & 5.50 & 8.90 & 26.09 & 9.76 & 25.60 & 5.03 & 40.40 & 11.40 & 27.90 & 3.80 & 43.20 & 12.50 & 27.90 & 5.34 & 45.70 & 12.50 & 34.60 & 6.90 & 54.00 & 100.0 & 100.0 & 100.0 & 300.0 \\
\hline $\begin{array}{l}\text { Albizzia chinensis } \\
\text { (Osbeck) Merr. }\end{array}$ & Fabaceae & - & - & - & - & 7.33 & 5.89 & 3.44 & 16.56 & - & - & - & - & 9.45 & 6.35 & 3.24 & 18.89 & - & - & - & - & - & - & - & - \\
\hline $\begin{array}{l}\text { Albizzia lebbeck } \\
\text { (L.) Benth. }\end{array}$ & Fabaceae & - & - & - & - & - & - & - & - & - & - & - & - & - & - & - & - & 6.30 & 2.60 & 3.20 & 12.00 & - & - & - & - \\
\hline $\begin{array}{c}\text { Anogeissus } \\
\text { latifolia (Roxb. ex } \\
\text { DC.) Wall. ex } \\
\text { Bedd. }\end{array}$ & Combretaceae & 6.30 & 3.90 & 6.90 & 17.10 & 2.40 & 2.50 & 6.40 & 11.30 & 5.70 & 5.08 & 5.50 & 17.00 & & & & & 6.30 & 3.90 & 5.70 & 15.80 & - & - & - & - \\
\hline $\begin{array}{c}\text { Bauhinia } \\
\text { variegata Linn. }\end{array}$ & Caesalpiniaceae & 12.50 & 7.10 & 5.80 & 25.40 & - & - & - & - & 5.70 & 3.50 & 2.80 & 12.05 & 6.34 & 2.55 & 2.67 & 11.55 & - & - & - & - & - & - & - & - \\
\hline $\begin{array}{l}\text { Bombax ceiba } \\
\text { Linn. }\end{array}$ & Malvaceae & - & - & - & - & 9.80 & 5.80 & 10.4 & 25.9 & 2.89 & 1.20 & 5.78 & 9.68 & 12.50 & 6.31 & 6.95 & 25.68 & 12.45 & 5.10 & 11.80 & 29.43 & - & - & - & - \\
\hline $\begin{array}{c}\text { Butea } \\
\text { monosperma } \\
\text { (Lam.) Taub. }\end{array}$ & Fabaceae & - & - & - & - & - & - & - & - & 2.89 & 2.28 & 10.74 & 15.60 & - & - & - & - & - & - & - & - & - & - & - & - \\
\hline $\begin{array}{l}\text { Cassia fistula } \\
\text { Linn. }\end{array}$ & Fabaceae & 6.30 & 6.30 & 3.80 & 16.40 & 4.90 & 2.50 & 7.45 & 15.09 & 5.70 & 2.29 & 2.50 & 10.50 & - & - & - & - & 3.10 & 1.34 & 4.14 & 8.50 & - & - & - & - \\
\hline $\begin{array}{l}\text { Dalbergia sissoo } \\
\text { Roxb. ex DC. }\end{array}$ & Fabaceae & 6.30 & 6.30 & 9.50 & 22.10 & 2.40 & 1.69 & 3.30 & 7.40 & 11.40 & 10.50 & 4.67 & 26.60 & 6.34 & 2.50 & 6.14 & 14.99 & 12.56 & 26.89 & 8.13 & 47.53 & - & - & - & - \\
\hline $\begin{array}{c}\text { Diospyros } \\
\text { montana Roxb. }\end{array}$ & Ebenaceae & - & - & - & - & 2.40 & 1.69 & 7.67 & 11.67 & - & - & - & - & - & - & - & - & - & - & - & - & - & - & - & - \\
\hline $\begin{array}{l}\text { Emblica officinalis } \\
\text { Linn. }\end{array}$ & Euphorbiaceae & 9.40 & 9.50 & 3.40 & 22.20 & - & - & - & - & 11.39 & 8.09 & 1.50 & 21.14 & 6.34 & 6.34 & 1.40 & 14.0 & 6.30 & 3.79 & 2.89 & 13.01 & - & - & - & - \\
\hline $\begin{array}{c}\text { Ficus glomerata } \\
\text { Roxb. }\end{array}$ & Moraceae & - & - & - & - & - & - & - & - & & - & - & - & - & - & - & - & 6.30 & 2.56 & 11.89 & 20.67 & - & - & - & - \\
\hline $\begin{array}{l}\text { Flacourtia indica } \\
\text { (Burm. f.) Merr. }\end{array}$ & Salicaceae & - & - & - & - & 7.30 & 6.56 & 1.89 & 15.78 & 2.86 & 2.30 & 1.30 & 6.47 & 6.25 & 3.80 & 3.04 & 13.09 & 3.12 & 1.28 & 1.92 & 6.32 & - & - & - & - \\
\hline $\begin{array}{l}\text { Grewia optiva J. } \\
\text { R. Drumm. }\end{array}$ & Tiliaceae & - & - & - & - & 4.88 & 3.31 & 1.78 & 9.96 & 2.86 & 1.16 & 6.30 & 10.32 & 3.12 & 1.27 & 6.01 & 10.40 & 6.25 & 2.56 & 5.49 & 14.30 & - & - & - & - \\
\hline $\begin{array}{c}\text { Holoptelea } \\
\text { integrifolia } \\
\text { (Roxb.) }\end{array}$ & Ulmaceae & - & - & - & - & - & - & - & - & - & - & - & - & - & - & - & - & 3.12 & 1.28 & 4.70 & 9.11 & - & - & - & - \\
\hline $\begin{array}{c}\text { Lannea } \\
\text { coromandelica } \\
\text { (Houtt.) Merr. }\end{array}$ & Anacardiaceae & 9.37 & 11.02 & 7.77 & 28.17 & - & - & - & - & 2.86 & 6.98 & 6.48 & 16.32 & - & - & - & - & - & - & - & - & - & - & - & - \\
\hline $\begin{array}{c}\text { Limonia } \\
\text { acidissima Linn. }\end{array}$ & Rutaceae & - & - & - & - & 2.44 & 2.48 & 2.20 & 7.12 & - & - & - & - & - & - & - & - & - & - & - & - & - & - & - & - \\
\hline $\begin{array}{c}\text { Mallotus } \\
\text { philippensis } \\
\text { (Lamk) Muell. } \\
\text { Arg. }\end{array}$ & Euphorbiaceae & 6.25 & 3.94 & 6.63 & 16.82 & 9.76 & 9.91 & 4.79 & 24.46 & 8.57 & 5.81 & 4.39 & 18.77 & 6.25 & 3.80 & 5.56 & 15.61 & 6.25 & 3.85 & 4.80 & 14.90 & - & - & - & - \\
\hline $\begin{array}{c}\begin{array}{c}\text { Ougeinia } \\
\text { oojeinensis } \\
\text { (Roxb.) Hochr. }\end{array}\end{array}$ & Fabaceae & 3.12 & 1.57 & 4.14 & 8.84 & 9.76 & 3.31 & 3.69 & 16.76 & - & - & - & - & - & - & - & - & - & - & - & - & - & - & - & - \\
\hline Phoenix sylvestris & Arecaceae & - & - & - & - & - & - & - & - & 2.86 & 4.65 & 6.67 & 14.18 & 3.12 & 1.27 & 2.88 & 7.27 & - & - & - & - & - & - & - & - \\
\hline
\end{tabular}




\section{Int.J.Curr.Microbiol.App.Sci (2018) 7(2): 2000-2009}

\begin{tabular}{|c|c|c|c|c|c|c|c|c|c|c|c|c|c|c|c|c|c|c|c|c|c|c|c|c|c|}
\hline (L.) Roxb. & & & & & & & & & & & & & & & & & & & & & & & & & \\
\hline $\begin{array}{c}\text { Pinus roxburghii } \\
\text { Sarg. }\end{array}$ & Pinaceae & 3.12 & 0.79 & 19.52 & 23.43 & 7.32 & 16.53 & 13.38 & 37.22 & 8.57 & 6.98 & 17.21 & 32.76 & 12.5 & 31.64 & 25.73 & 69.87 & 6.25 & 6.41 & 16.87 & 29.53 & - & - & - & - \\
\hline $\begin{array}{l}\text { Pyrus pashia } \\
\text { Buch. Ham. } \\
\text { D.Don }\end{array}$ & Rosaceae & - & - & - & - & 7.32 & 5.79 & 2.18 & 15.28 & 5.71 & 3.49 & 2.32 & 11.52 & 6.25 & 2.53 & 2.21 & 10.99 & 3.12 & 1.28 & 4.37 & 8.78 & - & - & - & - \\
\hline $\begin{array}{l}\text { Shorea robusta } \\
\text { Gaertn. f. }\end{array}$ & Dipterocarpaceae & 12.5 & 36.22 & 10.62 & 59.33 & - & - & - & - & - & - & - & - & - & - & - & - & - & - & - & - & - & - & - & - \\
\hline $\begin{array}{l}\text { Syzygium cumini } \\
\text { (L.) Skeels }\end{array}$ & Myrtaceae & 6.25 & 3.94 & 3.87 & 14.06 & 2.44 & 0.83 & 5.30 & 8.57 & - & - & - & - & - & - & - & - & 3.12 & 1.28 & 4.09 & 8.50 & & & & \\
\hline $\begin{array}{l}\text { Terminalia arjuna } \\
\text { Wight \& Arn. }\end{array}$ & Combretaceae & - & - & - & - & 2.44 & 0.83 & 12.65 & 15.92 & - & & - & - & - & - & - & - & - & - & - & - & - & - & - & - \\
\hline $\begin{array}{l}\text { Terminalia } \\
\text { bellerica (Gaertn.) } \\
\text { Roxb. }\end{array}$ & Combretaceae & - & - & - & - & - & - & - & - & 2.86 & 2.33 & 10.54 & 15.73 & 3.12 & 1.27 & 15.33 & 19.72 & - & - & - & - & - & - & - & - \\
\hline $\begin{array}{l}\text { Terminalia } \\
\text { chebula Retz. }\end{array}$ & Combretaceae & - & - & - & - & - & - & - & - & - & - & - & - & 3.12 & 1.27 & 11.77 & 16.16 & - & - & - & - & - & - & - & - \\
\hline $\begin{array}{c}\text { Terminalia } \\
\text { tomentosa Roxb. } \\
\text { (ex DC) Wight \& } \\
\text { Arn. }\end{array}$ & Combretaceae & 6.25 & 3.94 & 9.16 & 19.34 & - & - & - & - & - & - & - & - & - & - & - & - & - & - & - & - & - & - & - & - \\
\hline $\begin{array}{l}\text { Toona ciliata } \\
\text { M.Roem. }\end{array}$ & Meliaceae & - & - & - & - & 2.44 & 1.65 & 4.56 & 8.65 & 2.86 & 3.49 & 3.25 & 9.60 & 3.12 & 1.27 & 1.80 & 6.19 & 3.12 & 1.28 & 3.24 & 7.65 & - & - & - & - \\
\hline $\begin{array}{c}\text { Wendlandia } \\
\text { exserta (Roxb.) } \\
\text { DC. }\end{array}$ & Rubiaceae & - & - & - & - & 4.88 & 3.31 & 3.75 & 11.93 & 2.86 & 1.16 & 4.59 & 8.61 & - & - & - & - & - & - & - & - & - & - & - & - \\
\hline \multicolumn{26}{|c|}{ SHRUBS } \\
\hline $\begin{array}{c}\text { Adhatoda vasica } \\
\text { Linn. }\end{array}$ & Acanthaceae & - & - & - & - & - & - & - & - & 8.00 & 24.44 & 0.67 & 33.12 & 3.33 & 4.17 & 0.60 & 8.10 & 3.45 & 8.91 & 3.69 & 16.05 & 5.00 & 6.13 & 0.77 & 11.90 \\
\hline $\begin{array}{c}\text { Asparagus } \\
\text { adscendens } \text { Roxb. }\end{array}$ & Asparagaceae & - & - & - & - & 14.81 & 15.79 & 0.18 & 30.78 & 8.00 & 7.56 & 0.20 & 15.75 & 13.33 & 16.25 & 0.09 & 29.67 & 13.79 & 12.54 & 0.25 & 26.59 & 5.00 & 2.45 & 0.10 & 7.55 \\
\hline $\begin{array}{l}\text { Bauhinia vahlii } \\
\text { Wight \& Arn. }\end{array}$ & Caesalpiniaceae & 19.05 & 18.52 & 3.40 & 40.96 & - & - & - & - & - & - & - & - & - & - & - & - & - & - & - & - & 5.00 & 1.84 & 3.36 & 10.20 \\
\hline $\begin{array}{l}\text { Calotropis procera } \\
\text { W.T.Aiton }\end{array}$ & Apocynaceae & - & - & - & - & - & - & - & - & - & - & - & - & - & - & - & - & 3.45 & 1.32 & 4.48 & 9.25 & - & - & - & - \\
\hline $\begin{array}{l}\text { Carissa carandas } \\
\text { Linn. }\end{array}$ & Apocynaceae & 9.52 & 10.37 & 15.39 & 35.29 & 11.11 & 14.83 & 6.38 & 32.32 & 8.00 & 10.22 & 4.91 & 23.13 & 13.33 & 15.41 & 5.13 & 33.88 & 13.79 & 10.23 & 5.87 & 29.89 & 20.00 & 29.45 & 7.29 & 56.74 \\
\hline $\begin{array}{c}\text { Celastrus } \\
\text { paniculatus Willd. }\end{array}$ & Celastraceae & 4.76 & 1.48 & 7.40 & 13.64 & 3.70 & 1.44 & 37.70 & 42.84 & - & - & - & - & - & - & - & - & - & - & - & - & - & - & - & - \\
\hline $\begin{array}{c}\text { Clematis } \\
\text { acuminata DC. }\end{array}$ & Ranunculaceae & - & - & - & - & - & - & - & - & - & - & - & - & - & - & - & - & - & - & - & - & 5.00 & 1.23 & 0.3 & 6.56 \\
\hline $\begin{array}{c}\text { Cissampelos } \\
\text { pareira Linn. }\end{array}$ & Menispermaceae & 14.29 & 13.33 & 0.25 & 27.88 & 14.82 & 16.27 & 0.16 & 31.24 & 12.00 & 7.56 & 0.10 & 19.65 & 13.33 & 15.42 & 0.10 & 28.84 & 13.79 & 10.20 & 0.25 & 27.24 & 15.00 & 7.98 & 0.25 & 23.22 \\
\hline $\begin{array}{c}\text { Cryptolepis } \\
\text { buchanani Roem. } \\
\text { \& Schult. }\end{array}$ & Apocynaceae & - & - & - & - & - & - & - & - & 16.00 & 7.11 & 2.86 & 25.97 & - & - & - & - & - & - & - & - & - & - & - & - \\
\hline $\begin{array}{c}\text { Desmodium } \\
\text { pulchellum (L.) } \\
\text { Benth. }\end{array}$ & Fabaceae & 9.52 & 7.41 & 12.58 & 29.51 & - & - & - & - & - & - & - & - & - & - & - & - & - & - & - & - & - & - & - & - \\
\hline $\begin{array}{l}\text { Dodonaea viscosa } \\
\text { (L.) Jacq. }\end{array}$ & Sapindaceae & - & - & - & - & - & - & - & - & 4.00 & 4.00 & 2.10 & 10.10 & 3.33 & 4.58 & 5.22 & 13.14 & - & - & - & - & 5.00 & 1.23 & 20.65 & 26.87 \\
\hline $\begin{array}{l}\text { Flemingia } \\
\text { fruticosa Wall. ex } \\
\text { Benth. }\end{array}$ & Fabaceae & - & - & - & - & - & - & - & - & 4.00 & 3.11 & 1.39 & 8.50 & - & - & - & - & - & - & - & - & - & - & - & - \\
\hline
\end{tabular}




\section{Int.J.Curr.Microbiol.App.Sci (2018) 7(2): 2000-2009}

\begin{tabular}{|c|c|c|c|c|c|c|c|c|c|c|c|c|c|c|c|c|c|c|c|c|c|c|c|c|c|}
\hline $\begin{array}{l}\text { Hamiltonia } \\
\text { suaveolens } \\
\text { (Roxb.) }\end{array}$ & Rubiaceae & - & - & - & - & 3.70 & 1.44 & 4.92 & 10.06 & - & - & - & - & - & - & - & - & 3.45 & 1.32 & 1.05 & 5.82 & - & - & - & - \\
\hline $\begin{array}{l}\text { Ipomea carnea } \\
\text { Jacq. }\end{array}$ & Convolvulaceae & - & - & - & - & - & - & - & - & - & 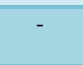 & - & - & - & - & - & - & - & - & - & - & 5.00 & 1.84 & 19.41 & 26.25 \\
\hline $\begin{array}{c}\text { Indigofera } \\
\text { pulchella Roxb. }\end{array}$ & Fabaceae & - & - & - & - & - & - & - & - & - & - & - & - & 3.33 & 1.67 & 3.79 & 8.79 & - & - & - & - & - & - & - & - \\
\hline $\begin{array}{c}\text { Lantana camara } \\
\text { Linn. }\end{array}$ & Verbenaceae & 4.76 & 3.70 & 4.39 & 12.86 & 3.70 & 5.74 & 7.03 & 16.47 & 4.00 & 4.00 & 2.09 & 10.09 & - & - & - & - & 10.34 & 13.86 & 7.01 & 31.22 & 10.00 & 4.29 & 9.94 & 24.24 \\
\hline $\begin{array}{c}\text { Lonicera japonica } \\
\text { Thunb. }\end{array}$ & Caprifoliaceae & - & - & - & - & - & - & - & - & 4.00 & 2.22 & 0.28 & 6.50 & - & - & - & - & - & - & - & - & - & - & - & - \\
\hline $\begin{array}{l}\text { Murraya koenigii } \\
\text { (L.) Spreng. }\end{array}$ & Rutaceae & 14.29 & 17.78 & 9.41 & 41.47 & 7.41 & 12.44 & 5.94 & 25.79 & 8.00 & 11.11 & 2.12 & 21.24 & 10.00 & 16.25 & 4.61 & 30.86 & 13.79 & 22.77 & 5.63 & 42.19 & 15.00 & 41.10 & 4.63 & 60.74 \\
\hline $\begin{array}{l}\text { Pogostemon } \\
\text { plectrantoides } \\
\text { Desf. }\end{array}$ & Lamiaceae & - & - & - & - & 7.41 & 7.18 & 1.47 & 16.05 & - & - & - & - & - & - & - & - & - & - & - & - & - & - & - & - \\
\hline $\begin{array}{c}\text { Randia } \\
\text { dumetorum Lam. }\end{array}$ & Rubiaceae & 9.52 & 14.81 & 32.95 & 57.29 & - & - & - & - & 4.00 & 4.44 & 40.09 & 48.53 & - & - & - & - & - & - & - & - & - & - & - & - \\
\hline $\begin{array}{l}\text { Randia spinosa } \\
\text { (Thunb.) Poir }\end{array}$ & Rubiaceae & - & - & - & - & - & - & - & - & - & - & - & - & 3.33 & 1.67 & 18.42 & 23.42 & - & - & - & - & - & - & - & - \\
\hline $\begin{array}{l}\text { Roylea cinerea (D. } \\
\text { Don) Baill. }\end{array}$ & Lamiaceae & - & - & - & - & 3.70 & 4.79 & 0.25 & 8.74 & - & - & - & - & - & - & - & - & 3.45 & 3.30 & 0.43 & 7.18 & - & - & - & - \\
\hline $\begin{array}{l}\text { Rubus elliptica } \\
\text { Sm. }\end{array}$ & Rosaceae & - & - & - & - & - & - & - & - & - & - & - & - & 6.67 & 2.5 & 22.72 & 31.88 & - & - & - & - & - & - & - & - \\
\hline $\begin{array}{l}\text { Vallaris heynei } \\
\text { (Roth) Kuntze }\end{array}$ & Apocynaceae & - & - & - & - & 7.41 & 4.31 & 0.53 & 12.25 & 4.00 & 2.67 & 0.07 & 6.74 & 10.00 & 7.92 & 0.29 & 18.21 & - & - & - & - & - & - & - & - \\
\hline $\begin{array}{l}\text { Vitex negundo } \\
\text { Linn. }\end{array}$ & Verbenaceae & - & - & - & - & - & - & - & - & 4.00 & 0.89 & 7.65 & 12.54 & - & - & - & - & - & - & - & - & - & - & - & - \\
\hline $\begin{array}{l}\text { Vitis parviflora } \\
\text { Baker }\end{array}$ & Vitaceae & - & - & - & - & - & - & - & - & - & - & - & - & 6.67 & 3.75 & 0.90 & 11.31 & - & - & - & - & - & - & - & - \\
\hline $\begin{array}{c}\text { Woodfordia } \\
\text { fruticosa }(\mathrm{L} .) \\
\text { Kurz }\end{array}$ & Lythraceae & 14.29 & 12.59 & 14.23 & 41.11 & 11.11 & 7.18 & 15.12 & 33.42 & 4.00 & 2.22 & 6.35 & 12.57 & 3.33 & 1.25 & 16.42 & 20.99 & 10.35 & 4.29 & 19.90 & 34.54 & - & - & - & - \\
\hline $\begin{array}{c}\text { Ziziphus } \\
\text { mauritiana Lam. }\end{array}$ & Rhamnaceae & - & - & - & - & 11.11 & 8.61 & 20.33 & 40.05 & - & 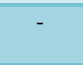 & - & - & - & - & - & - & 10.34 & 8.25 & 51.44 & 70.03 & 5.00 & 1.23 & 15.66 & 21.89 \\
\hline $\begin{array}{l}\text { Ziziphus oxyphylla } \\
\text { Edgew. }\end{array}$ & Rhamnaceae & - & - & - & - & - & - & - & - & 8.00 & 8.44 & 29.12 & 45.57 & 10.00 & 9.17 & 21.72 & 40.89 & - & - & - & - & - & - & - & - \\
\hline \multicolumn{26}{|c|}{ HERBS } \\
\hline $\begin{array}{l}\text { Achyranthes } \\
\text { aspera Linn. }\end{array}$ & Amaranthaceae & 21.05 & 28.25 & 0.39 & 49.69 & - & - & - & - & 21.05 & 23.08 & 5.86 & 49.99 & 30.00 & 17.16 & 8.57 & 55.73 & 6.67 & 11.67 & 1.09 & 19.43 & 18.75 & 18.47 & 0.94 & 38.16 \\
\hline $\begin{array}{c}\text { Ageratum } \\
\text { conyzoides Linn. }\end{array}$ & Asteraceae & - & - & - & - & - & - & - & - & 10.53 & 20.07 & 2.83 & 33.42 & 10.00 & 11.83 & 13.44 & 35.27 & - & - & - & - & 6.25 & 17.19 & 3.09 & 26.54 \\
\hline $\begin{array}{l}\text { Anisomeles indica } \\
\text { (L.) Kuntze }\end{array}$ & Lamiaceae & - & - & - & - & - & - & - & - & - & & - & - & - & - & - & - & 6.67 & 3.89 & 7.88 & 18.44 & - & - & - & - \\
\hline $\begin{array}{l}\text { Abrus precatorius } \\
\text { Linn. }\end{array}$ & Leguminaceae & - & - & - & - & - & - & - & - & - & & - & - & - & - & - & - & - & - & - & - & 6.25 & 1.91 & 0.94 & 9.10 \\
\hline $\begin{array}{l}\text { Artemisia vulgaris } \\
\text { Linn. }\end{array}$ & Asteraceae & - & - & - & - & - & - & - & - & 15.79 & 19.40 & 20.03 & 55.22 & - & - & - & - & - & - & - & - & - & - & - & - \\
\hline $\begin{array}{c}\text { Barleria cristata } \\
\text { Linn. }\end{array}$ & Acanthaceae & - & - & - & - & 12.5 & 20.46 & 13.24 & 46.19 & - & - & - & - & - & - & - & - & - & - & - & - & - & - & - & - \\
\hline $\begin{array}{l}\text { Bidens pilosa } \\
\text { Linn. }\end{array}$ & Asteraceae & 15.79 & 33.18 & 4.23 & 53.21 & 37.5 & 50.76 & 34.68 & 122.94 & 10.53 & 15.05 & 16.36 & 41.93 & 20.00 & 18.94 & 48.61 & 87.54 & 13.33 & 12.78 & 23.69 & 49.80 & 6.25 & 14.01 & 14.18 & 34.44 \\
\hline $\begin{array}{l}\text { Boerrhaevia } \\
\text { diffusa Linn. }\end{array}$ & Nyctaginaceae & - & - & - & - & - & - & - & - & - & - & - & - & - & - & - & - & - & - & - & - & 6.25 & 1.91 & 3.36 & 11.52 \\
\hline
\end{tabular}




\section{Int.J.Curr.Microbiol.App.Sci (2018) 7(2): 2000-2009}

\begin{tabular}{|c|c|c|c|c|c|c|c|c|c|c|c|c|c|c|c|c|c|c|c|c|c|c|c|c|c|}
\hline $\begin{array}{c}\text { Cassia mimosoides } \\
\text { Linn. }\end{array}$ & Caesalpiniaceae & - & - & - & - & - & - & - & - & 15.79 & 3.68 & 2.24 & 21.71 & - & - & - & - & - & - & - & - & - & - & - & - \\
\hline Cassia tora Linn. & Caesalpiniaceae & 10.53 & 9.86 & 3.02 & 23.41 & - & - & - & - & - & - & - & - & - & - & - & - & 6.67 & 11.11 & 22.30 & 40.07 & 6.25 & 6.37 & 6.13 & 18.75 \\
\hline $\begin{array}{c}\text { Cynoglossum } \\
\text { wallichi G. Don }\end{array}$ & Boraginaceae & 5.26 & 5.83 & 16.09 & 27.19 & - & - & - & - & - & - & - & - & - & - & - & - & - & - & - & - & - & - & - & - \\
\hline $\begin{array}{l}\text { Chenopodium } \\
\text { album Linn. }\end{array}$ & Chenopodiaceae & - & - & - & - & - & - & - & - & - & - & - & - & - & - & - & - & - & - & - & - & 6.25 & 3.18 & 5.49 & 14.92 \\
\hline $\begin{array}{c}\text { Chenopodium } \\
\text { ambrosoides Linn. }\end{array}$ & Chenopodiaceae & - & - & - & - & - & - & - & - & - & - & - & - & - & - & - & - & - & - & - & - & 6.25 & 2.54 & 3.78 & 12.57 \\
\hline $\begin{array}{c}\text { Dioscorea sativa } \\
\text { Thunb. }\end{array}$ & Dioscoreaceae & - & - & - & - & - & - & - & - & 5.26 & 1.34 & 14.53 & 21.13 & - & - & - & - & - & - & - & - & - & - & - & - \\
\hline $\begin{array}{l}\text { Erua scandens } \\
\text { Mart. }\end{array}$ & Amaranthaceae & - & - & - & - & - & - & - & - & - & - & - & - & - & - & - & - & 20.00 & 12.22 & 2.36 & 34.58 & - & - & - & - \\
\hline $\begin{array}{l}\text { Herpetospermum } \\
\text { pedunculosum } \\
\text { Ser. C.B. Clarke }\end{array}$ & Cucurbitaceae & - & - & - & - & - & - & - & - & - & - & - & - & - & - & - & - & - & - & - & - & 6.25 & 1.27 & 8.59 & 16.12 \\
\hline $\begin{array}{l}\text { Leucas lanata } \\
\text { Benth. }\end{array}$ & Lamiaceae & - & - & - & - & - & - & - & - & 10.53 & 4.01 & 6.21 & 20.75 & - & - & - & - & - & - & - & - & & & & \\
\hline $\begin{array}{l}\text { Marsdenia } \\
\text { tenacissima } \\
\text { (Roxb.) }\end{array}$ & Apocynaceae & - & - & - & - & - & - & - & - & - & - & - & - & - & - & - & - & - & - & - & - & 6.25 & 1.91 & 13.47 & 21.63 \\
\hline $\begin{array}{l}\text { Parthenium } \\
\text { hysterophorus } \\
\text { Linn. }\end{array}$ & Asteraceae & - & - & - & - & 12.5 & 15.15 & 45.88 & 73.53 & 10.53 & 13.38 & 31.98 & 55.88 & - & - & - & - & 26.67 & 38.33 & 36.71 & 101.71 & 6.25 & 11.46 & 17.10 & 34.82 \\
\hline $\begin{array}{l}\text { Phlogancanthus } \\
\text { pubinervius T. } \\
\text { Anders. }\end{array}$ & Acanthaceae & - & - & - & - & - & - & - & - & - & - & - & - & - & - & - & - & - & - & - & - & 6.25 & 1.91 & 20.44 & 28.60 \\
\hline $\begin{array}{l}\text { Phlomis bracteosa } \\
\text { Royle ex Benth. }\end{array}$ & Lamiaceae & 10.53 & 5.38 & 12.22 & 28.12 & - & - & - & - & - & - & - & - & - & - & - & - & - & - & - & - & - & - & - & - \\
\hline $\begin{array}{c}\text { Polygonum } \\
\text { hydropiper Linn. }\end{array}$ & Polygonaceae & - & - & - & - & - & - & - & - & - & - & - & - & 10.00 & 20.71 & 11.44 & 42.16 & - & - & - & - & - & - & - & - \\
\hline $\begin{array}{l}\text { Rubus niveus } \\
\text { Thunb. }\end{array}$ & Rosaceae & 5.26 & 1.35 & 29.41 & 36.02 & - & - & - & - & - & - & - & - & - & - & - & - & - & - & - & - & - & - & - & - \\
\hline $\begin{array}{c}\text { Siegesbeckia } \\
\text { orientalis Linn. }\end{array}$ & Asteraceae & 10.53 & 7.17 & 0.44 & 18.14 & - & - & - & - & - & - & - & - & - & - & - & - & - & - & - & - & - & - & - & - \\
\hline $\begin{array}{c}\text { Trifolium repens } \\
\text { Linn. }\end{array}$ & Fabaceae & - & - & - & - & - & - & - & - & - & - & - & - & 20.00 & 26.63 & 1.84 & 48.47 & - & - & - & - & 6.25 & 15.92 & 0.56 & 22.73 \\
\hline $\begin{array}{l}\text { Urena lobata } \\
\text { Linn. }\end{array}$ & Malvaceae & 10.53 & 4.93 & 3.98 & 19.44 & 37.5 & 13.64 & 6.21 & 57.34 & - & - & - & - & 10.00 & 4.73 & 16.11 & 30.85 & 20.00 & 10.00 & 5.97 & 35.97 & 6.25 & 1.91 & 1.92 & 10.08 \\
\hline $\begin{array}{c}\text { Xanthium } \\
\text { strumarium Linn. }\end{array}$ & Asteraceae & 10.53 & 4.04 & 30.20 & 44.77 & - & - & - & - & - & - & - & - & - & - & - & - & - & - & - & - & - & - & - & - \\
\hline
\end{tabular}


Table.2 Shannon Diversity Index in different A. catechu bearing forest types

\begin{tabular}{|c|c|c|c|}
\hline Forest Types & Trees & Shrubs & Herbs \\
\hline Dry Shiwalik Sal Forest & 0.85 & 0.68 & 0.45 \\
\hline Northern Dry Mixed Deciduous Forest & 0.94 & 0.70 & 0.26 \\
\hline Dry Deciduous Scrub Forest & 0.90 & 0.77 & 0.59 \\
\hline Lower Shiwalik Chirpine Forest & 0.79 & 0.81 & 0.57 \\
\hline Dry Riverine Forest & 0.84 & 0.83 & 0.54 \\
\hline Acacia catechu plantations & 0.00 & 0.64 & 0.65 \\
\hline CD $_{\mathbf{0 . 0 5}}$ & 0.10 & 0.17 & 0.30 \\
\hline
\end{tabular}

Table.3 Similarity index under different $A$. catechu bearing forest types

\begin{tabular}{|c|c|c|c|c|c|c|}
\hline Similarity & $\mathbf{F}_{\mathbf{2}}$ & $\mathbf{F}_{\mathbf{3}}$ & \multicolumn{5}{|c|}{$\mathbf{F}_{\mathbf{4}}$} & $\mathbf{F}_{\mathbf{5}}$ & $\mathbf{F}_{\mathbf{6}}$ \\
\hline $\mathbf{F}_{\mathbf{1}}$ & 69.57 & 81.81 & 54.54 & 76.19 & 15.38 \\
\hline $\mathbf{F}_{\mathbf{2}}$ & - & 100 & 86.96 & 123.81 & 11.11 \\
\hline $\mathbf{F}_{\mathbf{3}}$ & - & - & 130 & 109.09 & 11.11 \\
\hline $\mathbf{F}_{\mathbf{4}}$ & - & - & - & 110 & 13.33 \\
\hline $\mathbf{F}_{\mathbf{5}}$ & - & - & - & - & 12.5 \\
\hline & & \multicolumn{5}{|c|}{ Shrubs } \\
\hline $\mathbf{F}_{\mathbf{1}}$ & 80 & 66.67 & 44.44 & 58.82 & 66.67 \\
\hline $\mathbf{F}_{\mathbf{2}}$ & - & 66.67 & 63.16 & 120 & 82.35 \\
\hline $\mathbf{F}_{\mathbf{3}}$ & - & - & 94.74 & 80 & 70 \\
\hline $\mathbf{F}_{\mathbf{4}}$ & - & - & - & 66.67 & 70.59 \\
\hline $\mathbf{F}_{\mathbf{5}}$ & - & - & - & - & 106.67 \\
\hline & & & Herbs & \\
\hline $\mathbf{F}_{\mathbf{1}}$ & 33.33 & 25 & 50 & 61.54 & 44.44 \\
\hline $\mathbf{F}_{\mathbf{2}}$ & - & 54.54 & 75 & 66.67 & 57.14 \\
\hline $\mathbf{F}_{\mathbf{3}}$ & - & - & 72.73 & 42.86 & 76.92 \\
\hline $\mathbf{F}_{\mathbf{4}}$ & - & - & - & 53.33 & 71.43 \\
\hline $\mathbf{F}_{\mathbf{5}}$ & - & - & - & - & 53.33 \\
\hline
\end{tabular}

$\mathrm{F}_{1}$ - $\quad$ Dry Shivalik Sal Forest

$\mathrm{F}_{2}-\quad$ Northern Dry Mixed Deciduous Forest

$\mathrm{F}_{3}$ - $\quad$ Dry Deciduous Scrub Forest
$\mathrm{F}_{4}$ - Lower Shivalik Chirpine Forest

$\mathrm{F}_{5}$ - $\quad$ Dry Riverine Forest

$\mathrm{F}_{6}-$ A. catechu plantations
Similarity index deciphers the interrelatedness of each forest type and enables to understand the hidden process of succession of different forest types. Under different forest types (Table 3), in case of trees, the higher value of similarity index was observed under $\mathrm{F}_{4}$
(Lower Shiwalik Chirpine forest): $F_{3}$ (Dry Deciduous Scrub forest) followed by $F_{2}$ (Northern Dry Mixed Deciduous forest): $F_{5}$ (Dry Riverine forest) and $\mathrm{F}_{4}$ (Lower Shiwalik Chirpine forest): $F_{5}$ (Dry Riverine forest). In case of shrubs, the higher value was observed 
under $\mathrm{F}_{2}$ (Northern Dry Mixed Deciduous forest): $\mathrm{F}_{5}$ (Dry Riverine forest) followed by $\mathrm{F}_{5}$ (Dry Riverine forest): $\mathrm{F}_{6}$ (A. catechu plantations) and $\mathrm{F}_{3}$ (Dry Deciduous Scrub forest): $F_{4}$ (Lower Shiwalik Chirpine forest). While for herbs, the higher value was observed under $\mathrm{F}_{3}$ (Dry Deciduous Scrub forest): $\mathrm{F}_{6}$ (A. catechu plantations) followed by $F_{2}$ (Northern Dry Mixed Deciduous forest): $\mathrm{F}_{4}$ (Lower Shiwalik Chirpine forest) and $\mathrm{F}_{3}$ (Dry Deciduous Scrub forest): $\mathrm{F}_{4}$ (Lower Shiwalik Chirpine forest). Suyal et al., (2010) opined that close proximity results in high similarity index of vegetation. Chandra et al., (2010) studied vegetational diversity in Garhwal Himalaya and reported high similarity index between sites having similar environmental conditions. Kharkwal et al., (2005) concluded that the distribution and species richness pattern largely depend upon the altitude and climatic variables like rainfall, temperature and these parameters decrease with increase in elevation. Low similarity between different forest stands indicates the microclimatic variations and hence species composition.

\section{Acknowledgement}

The author would like to thank Department of Science and Technology, Ministry of Science and Technology, Government of India, for their financial assistance provided throughout during the course of this research work. The author also wishes to thank H.P. Forest Department personnel for their timely and valuable help.

\section{References}

Adhikari, B. S., Uniyal, S. K. and Rawat, G. S. 2009. Vegetation structure and community patterns of Tehri dam submergence zone, Uttarakhand, India. Eurasian Journal of Bioscience 3: 40-49.

Ahmad, I., Ahmad, M. S. A., Hussain, M.., Ashraf, M. Y., Ashraf and Hameed, M. 2010.
Spatiotemporal aspects of plant community structure in open scrub rangelands of sub mountainous Himalayan plateaus. Pakistan Journal of Botany. 42(5): 3431-3440.

Bhatt, V. P., and Purohit, V. K. 2009. Floristic structure and phytodiversity along an elevational gradient in Peepalkoti-Joshimath area of Garhwal Himalaya, India. Natural Science. 7(9): 63-74.

Champion, H. G., and Seth, S. K. 1968. A revised survey of the forest types of India. Manager of Publications, Delhi, India. 404p.

Chandra, J., Rawat, V. S., and Rawat, Y. S. and Ram, J. 2010. Vegetational diversity along an altitudinal range in Garhwal Himalaya. International Journal of Biodiversity and Conservation 2(1): 14-18.

Chauhan, N. S. 1999. Medicinal and aromatic plants of Himachal Pradesh. Indus Publishing Company. New Delhi, India. 632p.

Dangwal, L. R., Tajinder, S., Singh, A., and Sharma, A. 2012. Plant diversity assessment in relation to disturbances in subtropical chir pine forest of the Western Himalaya of district Rajouri, J\&K, India. International Journal of Plant, Animal \& Environmental Sciences 2(2): 206-213.

Gairola, S., Rawal, R. A., and Todaria, N. P. 2008. Forest vegetation patterns along an altitudinal gradient in sub-alpine zone of west Himalaya, India. African Journal of Plant Science 2(6): 42-48.

Gupta, B., Chauhan, P. S., and Dass, B. 2007. Allelopathic effect of leaf leachates of Pinus roxburghii Sargent on seeds of some grasses. Indian Forester 133(7): 997-1000.

Huang, W., Pohjonen, V., Johanson, S., Katigula, M. I. L., and Lukkanen, O. 2003. Species diversity, forest structure and species composition in Tanzania tropical forests. Forest Ecology and Management 173:11-24 http://dx.doi.org/10.1016/S037-1127(01)008 20-9.

Iqbal, K., Bhat, J. A., Pala, N. A., and Negi, A. K. 2012. Structure and composition estimation of plant species around Khoh river of Garhwal Himalaya. Journal of Biodiversity and Environmental Sciences 2(9): 1-11.

Kharkwal, G., Mehrotra, P., Rawat, Y. S., and Pangtey, Y. P. S. 2005. Phytodiversity and growth form in relation to altitudinal gradient 
in the central Himalayan (Kumaon) region of India. Current Science 89(5): 873-878.

Luna, R. K. 2005. Plantation trees. International Book Distributors, New Delhi, India. 975p.

Mueller, P. K. 2003. Nutrient relations in the herbaceous layer in deciduous forest ecosystem. In: Gillian F S and Roberts M R. (eds.). The herbaceous layer in forests of eastern North America. Oxford University Press, New York. pp 15-37.

Mukhia, P. K. 2011. Floristic composition and species diversity of the Chirpine forest ecosystem, Lobesa, Western Bhutan. Forestry Nepal. 1-4.

Odum, E. P. 1971. Fundamentals of Ecology. $3^{\text {rd }}$ Ed. W.B. Saunders Company. London. 574 pp.

Phillips, B. A. 1959. Methods of vegetation survey. Hevery Holt and Co. Inc.

Sorenson, T. 1948. A method of establishing group of equal amplitude in plant sociology based on similarity of the species content. Kongelige Danskse Videnskabernes Selskab Skrifter. Copenhagen 5(4): 1-34.

Sharma, C. M., Baduni, N. P., Gairola, S., Ghildiyal, S. K., and Suyal, S. 2010. Tree diversity and carbon stocks of some major forest types of Garhwal Himalaya, India. Forest Ecology and Management 260: 21702179.

Shukla, R. P. 2009. Patterns of plant species diversity across terai landscape in northeastern Uttar Pradesh, India. Tropical Ecology 50(1): 111-123.

Suyal, S., Sharma, C. M., Gairola, S., Ghildiyal,
S. K.. Rana, C. S., and Butola, D. S. 2010. Phytodiversity (angiosperms and gymnosperms) in Chaurangikhal forest of Garhwal Himalaya, Uttrakhand, India. Indian Journal of Science and Technology 3(3): 267-275.

Tadele, D., Lulekal, E., Damtie, D., and Assefa, A. 2014. Floristic diversity and regeneration status of woody plants in Zengena forest, a remnant montane forest patch in northwestern Ethiopia. Journal of Forestry Research 25: 329-336.

Tripathi, K. P., and Singh, B. 2009. Species diversity and vegetation structure across various strata in natural and plantation forests in Katerniaghat Wildlife Sanctuary, North India. Tropical Ecology 50(1): 191-200.

Yang, L., Liu, N., Ren, H., and Wang, J. 2009. Facilitation by two exotic Acacia: Acacia auriculiformis and Acacia mangium as nurse plants in south China. Forest Ecology and Management 257: 1786-1793.

Zegeye, H., Teketay, D., and Kelbessa, E. 2006. Diversity, regeneration status and socioeconomic importance of the vegetation in the islands of Lake Ziway, south-central Ethiopia. Flora 201: 483-498.

Zegeye, H., Teketay, D., and Kelbessa, E. 2011. Diversity and regeneration status of woody species in Tara Gedam and Abebaye Forests, northwestern Ethiopia. Journal of Forestry Research 22: 315-328.

\section{How to cite this article:}

Sanyam and Vipasha. 2018. Vegetation Structure, Floristic Composition and Species Diversity of Acacia catechu Bearing Forest Types in Western Himalaya, India. Int.J.Curr.Microbiol.App.Sci. 7(02): 2000-2009. doi: https://doi.org/10.20546/ijcmas.2018.702.239 